\title{
Deriving the exchange times for a model of trap-assisted tunnelling
}

\author{
Juraj Racko, Miroslav Mikolášek, \\ Magdaléna Kadlečíková, Peter Benko, Aleš Chvála ${ }^{1}$
}

\begin{abstract}
The work presents a physical model of trap-assisted tunnelling that allows assessing the impact of traps upon the total current through metal/semiconductor heterostructures. The model is based on expressing the occupation probability of the trapping centres by electrons in terms of thermal and tunnelling capture and emission times, commonly referred to as exchange times. The occupation probabilities calculated in this way are then used to evaluate the generation-recombination rates occurring in the continuity equations.

K e y w or d s: trap-assisted tunnelling, capture and emission probability, exchange times
\end{abstract}

\section{Introduction}

The need to explain and describe the charge transport in thin MOS and MIM structures has led to the development of various models of trap-assisted tunnelling (TAT). In the model of Chou et al [1] the trap states in the band gap of ultrathin oxides are generated during high field electrical stress. These traps behave as intermediate tunnelling sites. The stress-induced leakage current was modelled as a product of the density of traps at a given position in the band diagram and the probability of electron transmission through the traps at the same position, then integrated over the thickness of the oxide layer [2]. Jiménez-Molinos et al [3] presented a physical model based on the theory of multiphonon transitions between detrapped and trapped states and the only fitting parameters are the trap energy level and concentration, and the Huang-Rhys factor. A generalized trap-assisted tunnelling model was proposed in [4]. An effective tunnelling barrier of trapezoidal shape was considered, instead of the triangular barrier utilized in the conventional trap-assisted tunnelling (TAT) model. Ielmini et al [5-8] described inelastic TAT while takeing into account also the hopping conduction $[9,10]$. They derived the trapassisted current by integration along the dielectric thickness and energy. Many of the models had been implemented in simulators of semiconductor structures and devices. Among the numerous models, specific position belongs to those of TAT in semiconductor heterostructures [11-14].

\section{Band diagram and exchange processes}

Our TAT model was first published in [15, 16], however, without presenting in detail how the tunnelling and thermal exchange times had been derived. The model considers a metal/semiconductor heterostructure that may contain numerous deep trapping levels in the forbidden band of the semiconductor. Each of these levels has its specific density and effective cross-section of the trapping centres. The centre may capture and emit free charge carriers from/to the conduction and valence bands not only due to thermal exchange processes but also due to tunnelling. Trap-assisted tunnelling of free charge carriers has a higher probability in the case of multiphonon excitation of the deep traps. Due to the interaction of phonons with electrons at the trap the single deep level splits into a set of discrete levels. Their density is described by the multiphonon distribution function.

A trapping centre $\mathrm{T}$ is considered lying in the energy band gap of the semiconductor at coordinates $\varepsilon_{\mathrm{t}}$ and $x_{\mathrm{t}}$. In total, twelve charge transport mechanisms are taken into account, as shown in Fig. 1. Carrier transition currents $I_{1}, \ldots, I_{6}$ fill in an unoccupied trapping centre while carrier transition currents $I_{7}, \ldots, I_{12}$ are released from an occupied trapping centre.

\section{Thermal transition currents}

The electron thermal transition current $I_{1}$ flows from the conduction band into an unoccupied trapping centre. According to the kinetics of the recombination-generation processes [17], current $I_{1}$ is expressed as

$$
I_{1}\left(\varepsilon_{\mathrm{t}}, x_{\mathrm{t}}\right)=q\left(1-f_{\mathrm{t}}\left(\varepsilon_{\mathrm{t}}, x_{\mathrm{t}}\right)\right) v_{\mathrm{th}}^{\mathrm{e}} \sigma_{\mathrm{t}}^{\mathrm{e}}\left(x_{\mathrm{t}}\right)
$$

where $f_{\mathrm{t}}$ is the occupation probability of the trapping centre, $v_{\mathrm{th}}^{\mathrm{e}}$ and $\sigma_{\mathrm{t}}^{\mathrm{e}}$ are the electron thermal velocity and electron cross-section and

$$
n\left(x_{\mathrm{t}}\right)=N_{\mathrm{c}} \exp \left(\frac{E_{\mathrm{Fn}}\left(x_{\mathrm{t}}\right)-E_{\mathrm{c}}\left(x_{\mathrm{t}}\right)}{k T}\right)
$$

Slovak University of Technology, Ilkovičova 3, 81219 Bratislava, Slovakia, Corresponding author: juraj.racko@stuba.sk 


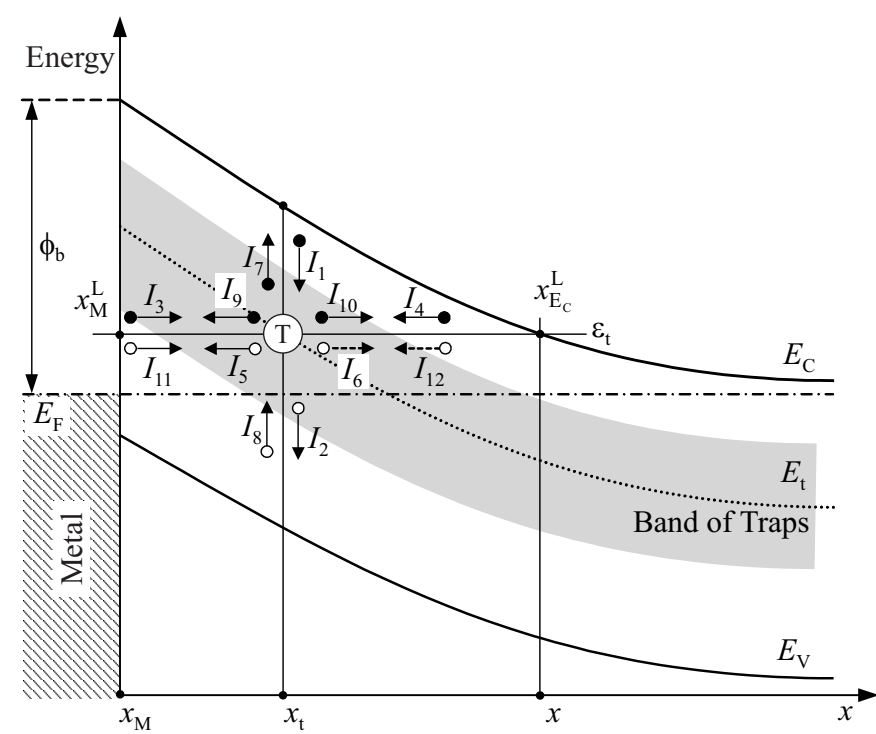

Fig. 1. Twelve exchange processes considered in the model of trap-assisted tunnelling in a metal-semiconductor structure.the arrows show the transitions of electrons or holes to or from the trap

is the electron concentration density in the conduction band at place $x_{\mathrm{t}}$ with quasi electron Fermi energy level $E_{\mathrm{F} n}$.

The electron thermal current $I_{7}$ flows in the opposite direction to current $I_{1}$. While current $I_{1}$ is proportional to $q\left(1-f_{\mathrm{t}}\left(\varepsilon_{\mathrm{t}}, x_{\mathrm{t}}\right)\right) v_{\mathrm{th}}^{\mathrm{e}} \sigma_{\mathrm{t}}^{\mathrm{e}}$, current $I_{7}$ must be proportional to $q f_{\mathrm{t}}\left(\varepsilon_{\mathrm{t}}\right) v_{\mathrm{th}}^{\mathrm{e}} \sigma_{\mathrm{t}}^{\mathrm{e}}$. In thermo-dynamic equilibrium (TDE) each pair of opposing currents must be equal, thus $I_{1}=$ $I_{7}, I_{2}=I_{8}, I_{3}=I_{9}, I_{4}=I_{10}, I_{5}=I_{11}$ and $I_{6}=I_{12}$. Then, from the equality of transition currents $I_{1}=I_{7}$ in TDE one receives

$$
\frac{1-f_{\mathrm{t}}\left(\varepsilon_{\mathrm{t}}, x_{\mathrm{t}}\right)}{f_{\mathrm{t}}\left(\varepsilon_{\mathrm{t}}\right)}=\frac{n_{\mathrm{t}}\left(\varepsilon_{\mathrm{t}}, x_{\mathrm{t}}\right)}{n\left(x_{\mathrm{t}}\right)}
$$

with an unknown density of thermally emitted electrons $n_{\mathrm{t}}$. It holds only in TDE that the occupation probability $f_{\mathrm{t}}$ is given by the Fermi-Dirac distribution function

$$
f_{\mathrm{t}}\left(\varepsilon_{\mathrm{t}}\right)=\frac{1}{1+\exp \left(\frac{\varepsilon_{\mathrm{t}}-E_{\mathrm{F}}}{k T}\right)},
$$

where $E_{\mathrm{F}}$ is the constant Fermi level. From (2) and (3) one calculates the unknown density of thermal emitted electrons $n_{\mathrm{t}}$ as

$$
n_{\mathrm{t}}\left(\varepsilon_{\mathrm{t}}, x_{\mathrm{t}}\right)=N_{\mathrm{c}} \exp \left(-\frac{E_{\mathrm{c}}\left(x_{\mathrm{t}}\right)-\varepsilon_{\mathrm{t}}}{k T}\right) .
$$

Then, the thermal transition current $I_{7}$ can be expressed as

$$
I_{7}\left(\varepsilon_{\mathrm{t}}, x_{\mathrm{t}}\right)=q f_{\mathrm{t}}\left(\varepsilon_{\mathrm{t}}, x_{\mathrm{t}}\right) v_{\mathrm{th}}^{\mathrm{e}} \sigma_{\mathrm{t}}^{\mathrm{e}} n_{\mathrm{t}}\left(\varepsilon_{\mathrm{t}}, x_{\mathrm{t}}\right) .
$$

The procedure is analogical in the case of holes. Therefore, we only briefly summarize the resulting formulae applicable for holes. The hole thermal current $I_{2}$ and the hole thermal current $I_{8}$ flowing in the opposite direction are expressed as

$$
\begin{gathered}
I_{2}\left(\varepsilon_{\mathrm{t}}, x_{\mathrm{t}}\right)=q\left(1-f_{\mathrm{t}}\left(\varepsilon_{\mathrm{t}}, x_{\mathrm{t}}\right) v_{\mathrm{th}}^{h} \sigma_{\mathrm{t}}^{h} p_{\mathrm{t}}\left(\varepsilon_{\mathrm{t}}, x_{\mathrm{t}}\right),\right. \\
I_{8}\left(x_{\mathrm{t}}\right)=q f_{\mathrm{t}}\left(\varepsilon_{\mathrm{t}} x_{\mathrm{t}}\right) v_{\mathrm{th}}^{\mathrm{h}} \sigma_{\mathrm{t}}^{\mathrm{h}} p\left(x_{\mathrm{t}}\right),
\end{gathered}
$$

where $v_{\mathrm{th}}^{\mathrm{h}}$ and $\sigma_{\mathrm{t}}^{\mathrm{h}}$ are the hole thermal velocity and hole cross-section and

$$
p_{\mathrm{t}}\left(\varepsilon_{\mathrm{t}}, x_{\mathrm{t}}\right)=N_{\mathrm{v}} \exp \left(\frac{E_{\mathrm{v}}\left(x_{\mathrm{t}}\right)-\varepsilon_{\mathrm{t}}}{k T}\right),
$$

is the density of thermally emitted holes from trapping level $\varepsilon_{\mathrm{t}}$ into the valence band $E_{\mathrm{v}}, E_{\mathrm{Fp}}$ is the hole quasi Fermi energy level and

$$
p\left(x_{\mathrm{t}}\right)=N_{\mathrm{v}} \exp \left(\frac{E_{\mathrm{v}}\left(x_{\mathrm{t}}\right)-E_{\mathrm{Fp}}\left(x_{\mathrm{t}}\right)}{k T}\right)
$$

is the hole concentration in the valence band at position $x_{\mathrm{t}}$.

\section{Tunnelling transition currents}

Let us focus on the electron tunnelling transition current flowing along the equipotential energy level $\varepsilon$ from the conduction band from points $x_{\mathrm{e}}^{*} \equiv x_{\mathrm{M}}^{\mathrm{L}}$ or $x_{E_{\mathrm{c}}}^{\mathrm{R}}$ (in Fig. 1, these points are intersections of energy level $\varepsilon$ with the metal $x_{\mathrm{M}}^{\mathrm{L}} \equiv x_{\mathrm{M}}$ or the bottom or the bottom edge of the conduction band $E_{\mathrm{c}}\left(x_{E_{\mathrm{c}}}^{\mathrm{R}}\right)=\varepsilon_{\mathrm{t}}$ into an unoccupied trapping centre at point $x_{\mathrm{t}}$. Generally, the electron tunnelling current density flowing through the potential barrier is expressed as a triple integral in $\mathbf{k}$-space [18]

$$
J \sim \frac{q}{(2 \pi)^{3}} \iiint 2 v_{x} \Gamma_{\mathrm{e}}\left(k_{x}\right) \mathrm{d} k_{x} \mathrm{~d} k_{y} \mathrm{~d} k_{z},
$$


where $k_{\mathrm{x}, \mathrm{y}, \mathrm{z}}$ are the cartesian wave numbers of tunnelling electrons, $\Gamma_{\mathrm{e}}\left(k_{x}\right)$ is the transmission coefficient of tunnelling electrons between points $x_{\mathrm{t}}$ and $x_{\mathrm{M}}^{\mathrm{L}}$ or $x_{E_{\mathrm{c}}}^{\mathrm{R}}$,

$$
v_{x}=\hbar k_{x} / m_{\mathrm{T}}^{\mathrm{e}}
$$

is the carrier velocity of tunnelling electrons in the direction of transport and $m_{\mathrm{T}}^{\mathrm{e}}$ is the electron tunnelling effective mass in the same direction. After multiplying (11) by the effective cross-section of the trap and taking into account the probability of non-occupation of the trap at point $x_{\mathrm{t}}$ and of the electron occurrence at point $x_{\mathrm{M}}^{\mathrm{L}}$ or $x_{E_{\mathrm{c}}}^{\mathrm{R}}$ the current density changes into current and assumes the form

$$
\begin{array}{r}
I_{3 \text { or } 4}=\frac{q \sigma_{\mathrm{t}}^{\mathrm{e}}}{(2 \pi)^{3}}\left(1-f_{\mathrm{t}}\left(x_{\mathrm{t}}\right)\right) f_{\mathrm{Fn}}\left(x_{\mathrm{e}}^{*}\right) \times \\
\times \iiint 2 v_{x} \Gamma_{\mathrm{e}}\left(k_{x}\right) \mathrm{d} k_{x} \mathrm{~d} k_{y} \mathrm{~d} k_{z},
\end{array}
$$

where $f_{\mathrm{Fn}}\left(x_{\mathrm{e}}^{*}\right)$ is the quasi electron Fermi-Dirac distribution function at points $x_{\mathrm{e}}^{*}=x_{\mathrm{M}}^{\mathrm{L}}$ or $x_{E_{\mathrm{c}}}^{\mathrm{R}}$.

Equation (12) will be simplified by transforming it from the cartesian wave number space into the energy space in a cylindrical coordination system. This is facilitated by the fact that the energy of tunnelling electrons assumes cylindrical symmetry in the direction of electron movement. For this purpose, transformation relations are used between the energy and wave numbers of electrons

$$
\begin{gathered}
k_{x}=\sqrt{\frac{2 m_{\mathrm{T}}^{\mathrm{e}} E_{x}}{\hbar^{2}}} \quad \mathrm{~d} k_{x}=\sqrt{\frac{m_{\mathrm{T}}^{\mathrm{e}}}{2 \hbar^{2} E_{x}}} \mathrm{~d} E_{x} \quad(13,1) \\
k_{y}=\sqrt{\frac{2 m_{\mathrm{R}}^{\mathrm{e}} E_{\perp}}{\hbar^{2}}} \cos \phi \quad k_{z}=\sqrt{\frac{2 m_{\mathrm{R}}^{\mathrm{e}} E_{\perp}}{\hbar^{2}}} \sin \phi \\
\rho=\sqrt{k_{y}^{2}+k_{z}^{2}}=\sqrt{\frac{2 m_{\mathrm{R}}^{\mathrm{e}} E_{\perp}}{\hbar^{2}}} \quad d \rho=\sqrt{\frac{m_{\mathrm{R}}^{\mathrm{e}}}{2 E_{\perp} \hbar^{2}}} \mathrm{~d} E_{\perp}
\end{gathered}
$$

The energy of the tunnelling electron consists of two components: $E_{x}=\frac{\hbar^{2} k_{x}^{2}}{2 m_{\mathrm{T}}^{\mathrm{e}}}$ is the electron energy in the direction of tunnelling transport, $E_{\perp}=\frac{\hbar^{2}\left(k_{y}^{2}+k_{z}^{2}\right)}{2 m_{\mathrm{R}}^{\mathrm{e}}}$ is the electron energy in the direction perpendicular to the tunnelling transport, and $m_{\mathrm{R}}^{\mathrm{e}}$ is the effective mass in this direction. In contrary to its two components, the total electron energy $\varepsilon_{\mathrm{t}}=E_{x}+E_{\perp}$ remains constant. If one component increases, the other component decreases by the same amount. Utilizing the transformation formula between cartesian and cylindrical coordinates

$$
\begin{aligned}
& \mathrm{d} k_{x} \mathrm{~d} k_{y} \mathrm{~d} k_{z}=\rho \mathrm{d} \rho \mathrm{d} \phi \mathrm{d} k_{x}= \\
& =\sqrt{\frac{m_{\mathrm{T}}^{\mathrm{e}}}{2 \hbar^{2} E_{x}}} \mathrm{~d} E_{x} \frac{m_{\mathrm{R}}^{\mathrm{e}} E_{\perp}}{\hbar^{2}} \mathrm{~d} E_{\perp} \mathrm{d} \phi
\end{aligned}
$$

the triple integral in $\boldsymbol{k}$-space is rewritten into the following form in the energy space

$$
\begin{aligned}
& \frac{q \sigma_{\mathrm{t}}^{\mathrm{e}}}{(2 \pi)^{3}} \iiint 2 v_{x} \Gamma_{\mathrm{e}} k_{x} \mathrm{~d} k_{x} \mathrm{~d} k_{y} \mathrm{~d} k_{z}= \\
& \frac{K_{\mathrm{e}}}{2} \int_{\varepsilon_{\mathrm{t}}}^{\varepsilon_{\mathrm{m}}} \Gamma_{\mathrm{e}}\left(E_{x}\right) \int_{0}^{\varepsilon_{\mathrm{t}}-E_{x}} \mathrm{~d} E_{\perp} \mathrm{d} E_{x} \int_{0}^{2 \pi} \mathrm{d} \phi
\end{aligned}
$$

where $K_{\mathrm{e}}=\frac{q m_{\mathrm{R}}^{\mathrm{e}} \sigma_{\mathrm{t}}^{\mathrm{e}}}{2 \pi^{3} \hbar^{3}}$. This expression can be further simplified. The inner integral over angle $\phi$ around axis $x$ gives the result $2 \pi$. Finally, the tunnelling probability $\Gamma_{\mathrm{e}}\left(k_{x}\right)$ depends only on component $\mathrm{Ex}$. Therefore, the integral over $\mathrm{d} E_{\perp}$ will be $E_{\perp}=\varepsilon_{\mathrm{t}}-E_{x}$ The maximum value of $E_{x}$ is equal to $\varepsilon_{\mathrm{t}}$, when the whole kinetic energy of the electron belongs to the direction of tunnelling and $E_{\perp}=0$. The lower value of $E_{x}=\varepsilon_{\mathrm{t}}-E_{\min }$ goes down to the bottom of the forbidden band. For $\varepsilon_{\mathrm{t}}-E_{\min }$ the tunnelling probability can be considered negligible. Then the last equation will be simplified as

$$
\begin{aligned}
& \frac{K_{\mathrm{e}}}{2} \int_{\varepsilon_{\mathrm{t}}}^{\varepsilon_{\mathrm{t}}-E_{\min }} \Gamma_{\mathrm{e}}\left(E_{x}\right) \int_{0}^{\varepsilon_{\mathrm{t}}-E_{x}} \mathrm{~d} E_{\perp} \mathrm{d} E_{x} \int_{0}^{2 \pi} \mathrm{d} \phi= \\
\varepsilon_{\mathrm{t}}-E_{\min } & K_{\mathrm{e}} \int_{\varepsilon_{\mathrm{t}}}^{2 \pi}\left(\varepsilon_{\mathrm{t}}-E_{x}\right) \Gamma_{e} E_{x} \mathrm{~d} E_{x} .
\end{aligned}
$$

For practical reasons the double integral will be replaced by summation, thus $\mathrm{d} E_{x} \rightarrow \Delta E_{x}$, and the electron energy component in the direction of tunnelling $E_{x}$ will be decremented from value $\varepsilon$ by $\Delta E_{x}$ in every summation step until the tunnelling probability assumes a negligible value. Thus,

$$
\begin{aligned}
& K_{\mathrm{e}} \int_{\varepsilon_{\mathrm{t}}}^{\varepsilon_{\mathrm{t}}-E_{\min }}\left(\varepsilon_{\mathrm{t}}-E_{x}\right) \Gamma_{\mathrm{e}}\left(E_{x}\right) \mathrm{d} E_{x} \cong \\
& \cong K_{\mathrm{e}} \sum_{j={ }^{\circ} 1}^{N} j \Delta E_{x}^{2} \Gamma_{\mathrm{e}}^{\mathrm{j}}\left(x_{\mathrm{e}}^{*}\right)
\end{aligned}
$$

where $N \cong \frac{\varepsilon_{\mathrm{t}}-E_{\mathrm{min}}}{\Delta E_{x}}$ and $\Gamma_{\mathrm{e}}^{\mathrm{j}}$ is the transmission coefficients for electron tunnelling. In WKB approximation one has for $x_{\mathrm{e}}^{*}=x_{\mathrm{M}}^{\mathrm{L}}$ or $x_{E_{\mathrm{c}}}^{\mathrm{R}}$

$$
\Gamma_{\mathrm{e}}^{\mathrm{j}}\left(x_{\mathrm{e}}^{*}\right)=\exp \left(-\frac{2}{\hbar} \int_{x_{\mathrm{t}}}^{x_{\mathrm{e}}^{*}} \sqrt{2 m_{\mathrm{T}}^{\mathrm{e}}\left(E_{\mathrm{c}}(x)-\varepsilon_{\mathrm{t}}+j \Delta E_{x}\right)} \mathrm{d} x\right)
$$

Now (12) can be rewritten into the final form adequately for $I_{3}$ or $I_{4}$

$$
I_{3,4}=K_{e}\left(1-f_{\mathrm{t}}\left(x_{\mathrm{t}}\right)\right) f_{\mathrm{Fn}}\left(x_{\mathrm{e}}^{*}\right) S_{\mathrm{e}}\left(x_{\mathrm{e}}^{*}\right)
$$


where $S_{\mathrm{e}}\left(x_{\mathrm{e}}^{*}\right)=\sum_{j=\circ}^{N} j \Delta E_{x}^{2} \Gamma_{\mathrm{e}}^{\mathrm{j}}\left(x_{\mathrm{e}}^{*}\right)$.

We proceed analogically when deriving electron currents $I_{9}$ or10 flowing out from the trap, thus in the opposite direction. The product of probabilities in (24) $\left(1-f_{\mathrm{t}}\left(x_{\mathrm{t}}\right)\right) f_{\mathrm{Fn}}\left(x_{\mathrm{e}}^{*}\right)$ is replaced by $f_{\mathrm{t}}\left(x_{\mathrm{t}}\right)\left(1-f_{\mathrm{Fn}}\left(x_{\mathrm{e}}^{*}\right)\right.$ for $I_{9}$ and $I_{10}$, respectively

$$
I_{9,10}=K_{\mathrm{e}} f_{\mathrm{t}}\left(x_{\mathrm{t}}\right)\left(1-f_{\mathrm{Fn}}\left(x_{\mathrm{e}}^{*}\right)\right) S_{\mathrm{e}}\left(x_{\mathrm{e}}^{*}\right)
$$

In the case of deriving the hole tunnelling currents the procedure is analogical and yields the relationships with $K_{h}=\frac{q m_{\mathrm{R}}^{\mathrm{h}} \sigma_{\mathrm{t}}^{\mathrm{h}}}{2 \pi^{3} \hbar^{3}}$ and $S_{h}\left(x_{\mathrm{h}}^{*}\right)=\sum_{j=\circ}^{N} j \Delta E_{x}^{2} \Gamma_{\mathrm{h}}^{\mathrm{j}}\left(x_{\mathrm{h}}^{*}\right)$

where $x_{\mathrm{h}}^{*}=x_{\mathrm{M}}^{\mathrm{L}}$ or $x_{\mathrm{Ev}}^{\mathrm{R}}$ (these points are intersections of energy level $\varepsilon$ with the metal $x_{\mathrm{M}}^{\mathrm{L}} \equiv x_{\mathrm{M}}$ or the bottom edge of the conduction band $\left.E_{\mathrm{v}}\left(x_{\mathrm{Ev}}^{\mathrm{R}}\right)=\varepsilon_{\mathrm{t}}\right)$

$$
\begin{gathered}
\left.I_{5,6}=K_{h} f_{\mathrm{t}}\left(x_{\mathrm{t}}\right)\left(1-f_{\mathrm{Fh}} x_{\mathrm{h}}^{*}\right)\right) S_{h}\left(x_{\mathrm{h}}^{*}\right) \\
I_{11,12}=K_{h}\left(1-f_{\mathrm{t}}\left(x_{\mathrm{t}}\right)\right) f_{\mathrm{Fh}}\left(x_{\mathrm{h}}^{*}\right) S_{h}\left(x_{\mathrm{h}}^{*}\right)
\end{gathered}
$$

where, $N \cong \frac{E_{\max }-\varepsilon_{\mathrm{t}}}{\Delta E_{x}}, f_{\mathrm{Fh}}$ is the Fermi-Dirac distribution function for the hole, $\Gamma_{\mathrm{h}}^{j}\left(x_{\mathrm{h}}^{*}\right)$ are the transmission coefficients of tunnelling holes between points $x_{\mathrm{t}}$ and $x_{\mathrm{h}}^{*}$. In WKB approximation one has

$$
\Gamma_{\mathrm{h}}^{j}\left(x_{\mathrm{e}}^{*}\right)=\exp \left(-\frac{2}{\hbar} \int_{x_{\mathrm{t}}}^{x_{\mathrm{h}}^{*}} \sqrt{2 m_{\mathrm{T}}^{\mathrm{h}}\left(\varepsilon_{\mathrm{t}}-E_{\mathrm{v}}(x)-j \Delta E_{x}\right)} \mathrm{d} x\right)
$$

If the intersections of energy level $\varepsilon$ with the upper edge of valence band $E_{\mathrm{v}}\left(x_{E_{\mathrm{v}}}^{\mathrm{R}}\right)=\varepsilon_{\mathrm{t}}$ do not exist, $I_{6}$ and $I_{12}$ are equal to zero.

\section{Exchange times}

The derived relations for calculating the thermal and tunnelling transition currents $I_{1} \ldots I_{12}$ contain an unknown occupation probability $f_{\mathrm{t}}$ (which is known only in TDE). Outside TDE, it does not hold anymore that in each pair the opposing currents are equal. Thus, (4) is no longer valid. Therefore, the occupation probability of trapping centre $f_{\mathrm{t}}$ is calculated as follows. It is obvious that the twelve transition currents must meet a conservation law like the current Kirchhoff law

$$
\sum_{i=1}^{6} I_{i}\left(\varepsilon_{\mathrm{t}}, x_{\mathrm{t}}\right)=\sum_{i=7}^{12} I_{i}\left(\varepsilon_{\mathrm{t}} x_{\mathrm{t}}\right)
$$

It is possible to extract $f_{\mathrm{t}}$ from this equation. To make this extraction transparent, a new quantity will be introduced, the exchange time $\tau$. The exchange times means the periods of time between the two successive transitions in each of the twelve exchange processes. The inverse value of the exchange time means the repetition frequency of the exchange process. We obtain these frequencies by dividing the transition currents $I_{1}\left(\varepsilon_{\mathrm{t}}, x_{\mathrm{t}}\right)$ to $I_{12}\left(\varepsilon_{\mathrm{t}}, x_{\mathrm{t}}\right)$ by unit charges and the terms containing the occupation probability $f_{\mathrm{t}}\left(\varepsilon_{\mathrm{t}}, x_{\mathrm{t}}\right)$,

$$
\begin{gathered}
\frac{1}{\tau_{(1 \ldots 6)}\left(\varepsilon, x_{\mathrm{t}}\right)}=\frac{I_{(1 \ldots 6)}\left(\varepsilon, x_{\mathrm{t}}\right)}{q\left(1-f_{\mathrm{t}}\left(\varepsilon, x_{\mathrm{t}}\right)\right)} \\
\tau_{(7 \ldots 12)}\left(\varepsilon, x_{\mathrm{t}}\right)=\frac{I_{(7 \ldots 12}\left(\varepsilon, x_{\mathrm{t}}\right)}{\left.q f_{\mathrm{t}}\left(\varepsilon, x_{\mathrm{t}}\right)\right)}
\end{gathered}
$$

Now the exchange times or frequencies are used to modify (29).

$$
\left(1-f_{\mathrm{t}}\left(\varepsilon, x_{\mathrm{t}}\right)\right) \sum_{i=1}^{6} \frac{1}{\tau_{i}\left(\varepsilon, x_{\mathrm{t}}\right)}=f_{\mathrm{t}} \sum_{i=7}^{12} \frac{1}{\tau_{i}\left(\varepsilon, x_{\mathrm{t}}\right)}
$$

From the above equations one can get the occupation probability $f_{\mathrm{t}}$ of the trapping centre by electrons and the non-occupation of the trapping centre - using only the exchange times $\tau_{1} \ldots \tau_{12}$ as

$$
\begin{aligned}
f_{\mathrm{t}}\left(\varepsilon, x_{\mathrm{t}}\right) & =\frac{\sum_{i=1}^{6} \tau_{i}\left(\varepsilon, x_{\mathrm{t}}\right)^{-1}}{\sum_{i=1}^{12} \tau_{i}\left(\varepsilon, x_{\mathrm{t}}\right)^{-1}} \\
1-f_{\mathrm{t}}\left(\varepsilon, x_{\mathrm{t}}\right) & =\frac{\sum_{i=7}^{12} \tau_{i}\left(\varepsilon, x_{\mathrm{t}}\right)^{-1}}{\sum_{i=1}^{12} \tau_{i}\left(\varepsilon, x_{\mathrm{t}}\right)^{-1}}
\end{aligned}
$$

The only input (fitting) parameters of the presented TAT model are electron and hole cross-sections.

\section{Density of trapping centres in the forbidden band}

Previous derivations were made for one trap lying anywhere in the forbidden band of the semiconductor. For practical solutions of the continuity and Poisson equations it is necessary to know also the density of trapping centres in the forbidden band.

Suppose trapping centres lying on the deep energy level $E_{\mathrm{t}}$ and let $N_{\mathrm{t}}\left(E_{\mathrm{t}}, x_{\mathrm{t}}\right)$ be the trap density belonging to this primary deep trapping level $E_{\mathrm{t}}$. The process of tunnelling of free electrons and holes via trapping centres is additionally supported by electron interaction with phonons. Instead of a discrete level $E_{\mathrm{t}}$ one must consider a band of energy levels. The probability of finding a trap above or below energy level $E_{\mathrm{t}}$ at $\left(\varepsilon_{\mathrm{t}}, x_{\mathrm{t}}\right)$ in the forbidden band is given by the multiphonon transition probability $M_{\mathrm{t}}$. The highest probability $M_{\mathrm{t}}$ belongs to those trapping centres that lie close to the middle of the band on the 
discrete level $E_{\mathrm{t}}$. The multiphonon transition probability is calculated as [19]

$$
\begin{gathered}
M_{\mathrm{t}}\left(\varepsilon_{\mathrm{t}}, x\right)=\frac{(\theta \mp S)^{2}}{\left(\theta^{2}+z^{2}\right)^{\frac{1}{4}}} \exp \left(\sqrt{z^{2}+\theta^{2}}\right. \\
-\theta \ln \left(\theta z^{-1}+\sqrt{1+\theta^{2} z^{-2}}-S\left(2 f_{\mathrm{B}}+1\right)-\frac{\hbar \omega_{0} \theta}{2 k T}\right)
\end{gathered}
$$

Here, $S$ is the Huang-Rhys factor representing the strength of electron-phonon coupling, $\hbar \omega_{0}$ is the effective phonon energy, $f_{\mathrm{B}}=1 /\left[\exp \left(\hbar \omega_{0} / k T\right)-1\right]$ is the Bose distribution function, and $\theta=\left|\varepsilon_{\mathrm{t}}-E_{\mathrm{D}, \mathrm{At}}\right| / \hbar \omega_{0}$ and $z=2 S \sqrt{f_{B}\left(1+f_{B}\right)}$. The sign inside the bracket in the nominator is negative for $E_{\mathrm{c}}-\varepsilon_{\mathrm{t}}>E_{\mathrm{c}}-E_{\mathrm{t}}$ and positive for $E_{\mathrm{c}}-\varepsilon_{\mathrm{t}}<E_{\mathrm{c}}-E_{\mathrm{t}}$.

The multiphonon density distribution of trapping centres $D_{\mathrm{t}}\left(\varepsilon_{\mathrm{t}}, x_{\mathrm{t}}\right)$ is expressed in terms of the multiphonon transition probability $M_{\mathrm{t}}$ as

$$
D_{\mathrm{t}}\left(\varepsilon_{\mathrm{t}}, x_{\mathrm{t}}\right)=\frac{N_{\mathrm{t}}\left(x_{\mathrm{t}}\right)}{\sqrt{2 \pi} S \hbar \omega_{0}} M_{\mathrm{t}}\left(E_{\mathrm{t}}, \varepsilon_{\mathrm{t}}, x_{\mathrm{t}}\right)
$$

Due to the multi-phonon transitions the amount of traps in the infinitesimally small energy interval $\mathrm{d} \varepsilon_{\mathrm{t}}$ at $\left(\varepsilon_{\mathrm{t}}, x_{\mathrm{t}}\right)$ is given as $D_{\mathrm{t}}\left(\varepsilon_{\mathrm{t}}, x_{\mathrm{t}}\right) \mathrm{d} \varepsilon_{\mathrm{t}}$. Nevertheless, several deep energy capture levels of donor or acceptor type may lie in the band gap of semiconductor. In such a case the formula of the density distribution of trapping centres must be extended by summing up all capture levels

$$
\begin{aligned}
& D_{\mathrm{t}}\left(\varepsilon_{\mathrm{t}}, x_{\mathrm{t}}\right)=D_{\mathrm{D}_{\mathrm{t}}}\left(\varepsilon_{\mathrm{t}}, x_{\mathrm{t}}\right)+D_{\mathrm{A}_{\mathrm{t}}}\left(\varepsilon_{\mathrm{t}}, x_{\mathrm{t}}\right)= \\
= & \frac{1}{\sqrt{2 \pi} S \hbar \omega_{0}}\left(\sum_{i} N_{\mathrm{D}_{\mathrm{t}}}^{i} M_{\mathrm{t}}\left(E_{\mathrm{D}_{\mathrm{t}}}^{i}, \varepsilon_{\mathrm{t}}, x_{\mathrm{t}}\right)+\right. \\
& \left.+\sum_{j} N_{\mathrm{A}_{\mathrm{t}}}^{j} M_{\mathrm{t}}\left(E_{\mathrm{A}_{\mathrm{t}}}^{j}, \varepsilon_{\mathrm{t}}, x_{\mathrm{t}}\right)\right)
\end{aligned}
$$

\section{Implementing the TAT model into fundamental semiconductor equations}

Knowing the probability and the density distribution of trapping centres one can calculate currents $I_{1} \ldots, I_{12}$. The output from the TAT model shall be considered in the fundamental semiconductor equations in three steps. We separate the contributions from thermal and tunnelling exchange processes.

First, at every point of the conduction band we can integrate contributions from all thermal electron or hole exchange processes. These are contributions from all traps lying along the vertical energy axis intersecting the conducting band at point $x=x_{\mathrm{t}}$. The result of this integration has a physical dimension $\mathrm{Am}^{-3}$ and for electrons is expressed as

$$
q U_{\mathrm{TAT}}^{\mathrm{e}}(x)=\int_{E_{\mathrm{v}}(x)}^{E_{\mathrm{c}}(x)}\left(I_{1}\left(\varepsilon_{\mathrm{t}}, x\right)-I_{7}\left(\varepsilon_{\mathrm{t}}, x\right)\right) D_{\mathrm{t}}\left(\varepsilon_{\mathrm{t}}, x\right) \mathrm{d} \varepsilon_{\mathrm{t}}
$$

and for holes as

$$
q U_{\mathrm{TAT}}^{\mathrm{h}}(x)=\int_{E_{\mathrm{v}}(x)}^{E_{\mathrm{c}}(x)}\left(I_{2}\left(\varepsilon_{\mathrm{t}}, x\right)-I_{8}\left(\varepsilon_{\mathrm{t}}, x\right)\right) D_{\mathrm{t}}\left(\varepsilon_{\mathrm{t}}, x\right) \mathrm{d} \varepsilon_{\mathrm{t}} .
$$

These equations extend the Shockley-Read-Hall generation-recombination model while neglecting the tunnelling exchange processes and limiting to the thermal exchange processes. These expressions are inserted in the right side of the electron continuity equation.

Second, in the case of tunnelling exchange processes the contributions are integrated from all electron or hole tunnelling exchange processes along the space $x$-axis at energy line $\varepsilon=\varepsilon_{\mathrm{t}}$. The result of this integration has a physical dimension $\mathrm{Am}^{-2}$ and is expressed as

$$
\begin{aligned}
& J_{\mathrm{TAT}}^{\mathrm{e}}(x)= \\
& =\int_{x_{\mathrm{M}}}^{x}\left(I_{4}\left(\varepsilon, x_{\mathrm{t}}\right)-I_{10}\left(\varepsilon, x_{\mathrm{t}}\right)\right) D_{\mathrm{t}}\left(\varepsilon, x_{\mathrm{t}}\right) \mathrm{d} x_{\mathrm{t}} .
\end{aligned}
$$

This expression is used if the band gap is bent up. In this case the hole trap assisted tunnelling current is zero, $J_{\text {TAT }}^{\mathrm{h}}(x)=0$. If that the band gap bent down the electron trap assisted tunnelling current is zero, $J_{\text {TAT }}^{\mathrm{e}}(x)=0$ and, now for holes it holds

$$
\begin{aligned}
& J_{\mathrm{TAT}}^{\mathrm{h}}(x)= \\
& =\int_{x_{\mathrm{M}}}^{x}\left(I_{5}\left(\varepsilon, x_{\mathrm{t}}\right)-I_{11}\left(\varepsilon, x_{\mathrm{t}}\right)\right) D_{\mathrm{t}}\left(\varepsilon, x_{\mathrm{t}}\right) \mathrm{d} x_{\mathrm{t}} .
\end{aligned}
$$

Equations $(37,38)$ express the trap-assisted-tunnelling current densities and are inserted in the left side of the electron and hole continuity equations. Equations (37) to (40) are now implemented in the differential electron continuity equation expressed as

$$
\frac{\mathrm{d}\left(J_{D}^{\mathrm{e}}(x)+J_{\mathrm{TAT}}^{\mathrm{e}}(x)\right)}{\mathrm{d} x}-q \frac{\partial n(x)}{\partial t}=q U_{\mathrm{TAT}}^{\mathrm{e}}(x)
$$

and in the differential hole continuity equation expressed in

$$
\frac{\mathrm{d}\left(J_{D}^{\mathrm{h}}(x)+J_{\mathrm{TAT}}^{\mathrm{h}}(x)\right)}{\mathrm{d} x}+q \frac{\partial p(x)}{\partial t}=-q U_{\mathrm{TAT}}^{\mathrm{h}}(x)
$$

where $J_{D}^{\mathrm{e}} \equiv J_{D}^{\mathrm{e}}(x)$ and $J_{D}^{\mathrm{h}} \equiv J_{D}^{\mathrm{h}}(x)$ are the electron and hole drift-diffusion currents.

Third and eventually, the probability of occupation of the trapping centres is used in the calculation of the concentration of deep ionized donors $N_{\mathrm{D}_{\mathrm{t}}}^{+}$and acceptors $N_{\mathrm{A}_{\mathrm{t}}}^{-}$These concentrations are calculated as 


$$
\begin{aligned}
& N_{\mathrm{D}_{\mathrm{t}}}^{+}(x)=\sum_{i} \int_{E_{\mathrm{V}}(x)}^{E_{\mathrm{C}}(x)}\left(1-f_{\mathrm{t}}\left(\varepsilon_{\mathrm{t}}\right)\right) D_{\mathrm{D}_{\mathrm{t}}}^{i}\left(E_{\mathrm{D}_{\mathrm{t}}}^{i}, \varepsilon_{\mathrm{t}}\right) \mathrm{d} \varepsilon_{\mathrm{t}}, \\
& N_{\mathrm{A}_{\mathrm{t}}}^{-}(x)=\sum_{j} \int_{E_{\mathrm{V}}(x)}^{E_{\mathrm{C}}(x)} f_{\mathrm{t}}\left(\varepsilon_{\mathrm{t}}\right) D_{\mathrm{A}_{\mathrm{t}}}^{j}\left(E_{\mathrm{A}_{\mathrm{t}}}^{j}, \varepsilon_{\mathrm{t}}\right) \mathrm{d} \varepsilon_{\mathrm{t}} .
\end{aligned}
$$

The Poisson equation can now be written in the usual form as

$$
\begin{gathered}
-\frac{\mathrm{d}}{\mathrm{d} x}\left(\kappa(x) \frac{\mathrm{d} \psi(x)}{\mathrm{d} x}+Q(x)\right)=q(p(x)-n(x)+ \\
\left.N_{\mathrm{D}}^{+}(x)-N_{\mathrm{A}}^{-}(x)+N_{\mathrm{D}_{\mathrm{t}}}^{+}(x)-N_{\mathrm{A}_{\mathrm{t}}}^{-}(x)\right)
\end{gathered}
$$

where $\kappa$ is the semiconductor permittivity, $Q$ is the sheet charge at the interface, $\psi$ is the electric potential, $N_{\mathrm{D}}^{+}$ and $N_{\mathrm{A}}^{-}$are the concentrations of shallow ionized donors and acceptors.

\section{Conclusion}

The concept of tunnelling exchange times presents a dominant contribution to the TAT model. The crucial problem in the TAT model is the calculation of the occupation probability. The new approach allows to simply calculate the probability of occupation of the trapping centres by free charge carriers and subsequently to get the thermal and tunnelling generation-recombination rates occurring in the continuity equations.

\section{Acknowledgements}

The work was supported by the Slovak Research and Development Agency under contracts No. APVV-170169 and No. APVV-15-0152, and by the scientific grant agency VEGA of the Ministry of Education, Science, Research and Sport of the Slovak Republic, projects VEGA 1/0532/19 and VEGA 1/0529/20.

\section{REFERENCES}

[1] A. I. Chou, K. Lai, K. Kumar, P. Chowdhury, and J. C. Lee, "Modeling of stress-induced leakage current in ultrathin oxides with the trap-assisted tunneling mechanism", Applied Physics Letters, vol. 70, no. 25, pp. 3407-3409, 1997.

[2] M. Herrmann and A. Schenk, "Field and hightemperature dependence of the long term charge loss in erasable programmable read only memories:Measurements and modeling", Journal of Applied Physics, vol. 77, no. 9, pp. 4522-4540, 1995.

[3] F. Jiménez-Molinos, F. Gámiz, A. Palma, P. Cartujo, and J. A. López-Villanueva, "Direct and trap-assisted elastic tunneling through ultrathin gate oxides", Journal of Applied Physics, vol. 91, no. 8, pp. 5116-5124, 2002.
[4] M. P. Houng, Y. H. Wang, and W. J. Chang, "Current transport mechanism in trapped oxides:A generalized trap-assisted tunneling model", Journal of Applied Physics, vol. 86, no. 3, pp. 1488-1491, 1999.

[5] D. Ielmini, A. S. Spinelli, M. A. Rigamonti, and A. L. Lacaita, "Modeling of SILC based on electron and hole tunneling I, Transient effects", IEEE Transactions on Electron Devices, vol. 47, no. 6, pp. 1258-1265, 2000.

[6] D. Ielmini, A. S. Spinelli, M. A. Rigamonti, and A. L. Lacaita, "Modeling of SILC based on electron and hole tunneling II, Steady-state", IEEE Transactions on Electron Devices, vol. 47, no. 6, pp. 1266-1272, 2000.

[7] D. Ielmini, A. S. Spinelli, A. L. Lacaita, A. Martinelli, and G. Ghidini, "A recombination- and trap-assisted tunneling model for stress-induced leakage current", Solid-State Electronics, vol. 45, no. 8, pp. 1361-1369, 2001.

[8] D. Ielmini, A. S. Spinelli, A. L. Lacaita, and G. Ghidini, "Modeling of stress-induced leakage current and impact ionization in MOS devices", Solid-State Electronics, vol. 46, no. 3, pp. 417-422, 2002.

[9] D. Ielmini, A. S. Spinelli, A. L. Lacaita, and A. Modelli, "A new two-trap tunneling model for the anomalous stress-induced leakage current (SILC) in Flash memories", Microelectronic Engineering, vol. 59, no. 1-4, pp. 189-195, 2001.

[10] D. Ielmini, A. S. Spinelli, A. L. Lacaita, and A. Modelli, "Modeling of anomalous SILC in flash memories based on tunneling at multiple defects", Solid-State Electronics, vol. 46, no. 11, pp. 1749-1756, 2002.

[11] D. M. Sathaiya and S. Karmalkar, "Thermionic trap-assisted tunneling model and its application to leakage current in nitrided oxides and AlGaNGaN high electron mobility transistors", Journal of Applied Physics, vol. 99, no. 9, p. 093701, 2006.

[12] Q. K. Yang, F. Fuchs, J. Schmitz, and W. Pletschen, "Investigation of trap-assisted tunneling current in InAs/ (GaIn) Sb superlattice long-wavelength photodiodes", Applied Physics Letters, vol. 81, no. 25, pp. 4757-4759, 2002.

[13] D. Rosenfeld and G. Bahir, "A model for the trap-assisted tunneling mechanism in diffused $\mathrm{n}-\mathrm{p}$ and implanted $\mathrm{n} / \mathrm{sup}$ pmp HgCdTe photodiodes", IEEE Transactions on Electron Devices, vol. 39, no. 7, pp. 1638-1645, 1992, DOI: 10.1109/16.141229.

[14] M. Zhang, Z. Huo, Z. Yu, J. Liu, and M. Liu, "Unification of three multiphonon trap-assisted tunneling mechanisms", Journal of Applied Physics, vol. 110, no. 11, p. 114108, 2011.

[15] J. Racko, P. Benko, I. Hotový, L. Harmatha, M. Mikolášek, R. Granzner, M. Kittler, F. Schwierz, and J. Breza, "A model of trap-assisted tunneling in GaN/AlGaN/GaN heterostructure based on exchange times", Applied Surface Science, vol. 312, pp. 68-73, 2014.

[16] J. Racko, P. Benko, M. Mikolášek, R. Granzner, M. Kittler, F. Schwierz, L. Harmatha, and J. Breza, "Simulation of real I-V characteristics of metal/GaN/AlGaN heterostructure based on the 12-EXT model of trap-assisted tunnelling", Applied Surface Science, vol. 395, pp. 122-130, 2017.

[17] A. Grove, Physics, and technology of semiconductor devices, N. York, J. Wiley, and Sons, 1967.

18] D. Schroeder Modelling of interface carrier transport for device simulation, Wien, Springer-Verlag, 2014.

[19] A. Schenk, "A model for the field and temperature dependence of Shockley-Read-Hall lifetimes in silicon", Solid-State Electronics, vol. 35, no. 11, pp. 1585-1596, 1992.

Received 9 December 2019 